\title{
Radar Emitter Identification under Transfer Learning and Online Learning
}

\author{
Yuntian Feng *, Yanjie Cheng, Guoliang Wang, Xiong Xu, Hui Han and Ruowu Wu \\ State Key Lab. of Complex Electromagnetic Environment Effects on Electronics and Information System, \\ Luoyang 471003, China; yanjiechengcemee@163.com (Y.C.); guoliangwangcemee@163.com (G.W.); \\ xiongxucemee@163.com (X.X.); huihancemee@163.com (H.H.); ruowuwucemee@163.com (R.W.) \\ * Correspondence: fengyuntian2009@live.cn
}

Received: 8 November 2019; Accepted: 20 December 2019; Published: 25 December 2019

\begin{abstract}
At present, there are two main problems in the commonly used radar emitter identification methods. First, when the distribution of training data and testing data is quite different, the identification accuracy is low. Second, the traditional identification methods usually include an offline training stage and online identifying stage, which cannot achieve the real-time identification of the radar emitter. Aimed at the above problems, this paper proposes a radar emitter identification method based on transfer learning and online learning. First, for the case where the target domain contains only a small number of labeled samples, the TrAdaBoost method is used as the basic learning framework to train a support vector machine, which can obtain useful knowledge from the source domain to aid in the identification of the target domain. Then, for the case where the target domain does not contain labeled samples, the Expectation-Maximization algorithm is used to filter the unlabeled samples in the target domain to generate the available training data. Finally, to make the identification quickly and accurately, we propose a radar emitter identification method, based on online learning to ensure real-time updating of the model. Simulation experiments show that the proposed method, based on transfer learning and online learning, has higher identification accuracy and good timeliness.
\end{abstract}

Keywords: transfer learning; online learning; radar emitter

\section{Introduction}

Radar emitter identification is the key link in radar reconnaissance. It extracts the characteristic parameters and working parameters on the basis of radar signal sorting. Based on these parameters, we can obtain information such as the system, use, type, and platform of the target radar, and further deduce the battlefield situation, threat level, activity rule, tactical intention, etc., and provide important intelligence support for its own decision-making [1].

Common radar emitter identification methods include feature matching based methods [2], intra-pulse analysis based methods [3], and machine learning based methods [4]. In the past battlefields, the types of radar emitters are simple and the number is limited, so the above methods can solve the problem of radar emitter identification well. The complex electromagnetic environment of the modern battlefield brings many difficulties to radar emitter identification, and poses two problems in the commonly used identification methods. First, when the distribution of training data and testing data is quite different, the identification accuracy is low and the traditional identification methods are unable to respond effectively to an unknown radar emitter; second, the above identification methods usually adopt two stages of offline training and online identification, which make the model training and update speed slow, which cannot realize the real-time identification of the radar emitter. 
In recent years, with the continuous development of machine learning technology, the transfer learning methods and the online learning methods have gained more and more attention. Transfer learning does not require that training data and testing data meet the condition of the same distribution in the model training process, and utilizes the knowledge of a large number of known samples for training, which is good for cross-domain learning. However, the transferring of a large amount of irrelevant information will also cause a negative transfer, which reduces the effect of identification. Online learning does not require the saving a large amount of training data, and can train the new classifier according to the latest samples and the current classifier, of which the training speed is fast and the identification accuracy is high.

In view of the characteristics of transfer learning and online learning, in order to meet the challenges brought by the complex electromagnetic environment of the modern battlefield, this paper studies radar emitter identification under transfer learning and online learning. First, for the case where the target domain contains only a small number of labeled samples, the TrAdaBoost method is used as the basic learning framework to train a support vector machine, which can obtain useful knowledge from the source domain to aid in the identification of the target domain. Then, for the case where the target domain does not contain labeled samples, the Expectation-Maximization algorithm is used to filter the unlabeled samples in the target domain to generate the available training data. Finally, to make the identification quickly and accurately, we propose a radar emitter identification method, based on online learning, to ensure real-time updating of the model.

Our major contributions are summarized as follows: (1) Focusing on the actual application scenarios to study radar emitter identification, and simultaneously solving the problem that the distribution of training data and testing data is quite different and the problem that the model cannot be updated in real-time, which provides a good way of thinking for future research in this area; (2) proposing the support vector machine model based on the TrAdaBoost method and transductive transfer learning, based on the EM algorithm for the radar emitter identification task, which can make fuller use of label information and sample information in the target domain; and (3) verifying the effectiveness of combining the transfer learning algorithms with the online learning algorithm, which helps to improve the speed of the transfer learning algorithms.

\section{Relevant Research}

\subsection{Transfer Learning}

Transfer learning refers to learning the knowledge in the source domain $D_{s}$, and using it in the target domain $D_{t}$, which does not have the same distribution as $D_{s}$, but is related to $D_{s}$, which makes good the problem of insufficient training data. Unlike traditional machine learning methods, transfer learning [5] does not require training data and testing data to satisfy the same-distribution hypothesis. It can discover and extract knowledge in the source domain $D_{s}$ that matches the distribution of the target domain $D_{t}$ and is useful for identification in the target domain $D_{t}$. Then, it establishes classification models in the target domain $D_{t}$, which can make efficient use of existing labeled samples to avoid re-labeling in the target domain $D_{t}$.

From the perspective of transfer methods, transfer learning includes four basic methods: Sample-based transfer, feature-based transfer, model-based transfer, and relationship-based transfer. The sample-based transfer method refers to producing rules according to certain weights and reusing data samples for transfer learning. The feature-based transfer method refers to mutual transfer by feature transformation, which reduces the gap between the source domain and the target domain, or transforms the data features of the source domain and the target domain into a unified feature space, and then utilizes the traditional machine learning methods for identification. The model-based transfer method refers to finding the parameter information shared between the source domain and the target domain in order to implement transferring. The relationship-based transfer method has a completely 
different approach from the above three methods, and focuses on the similarity between the source domain samples and target domain samples.

\subsection{Online Learning}

In the field of artificial intelligence and machine learning, in order to solve the problem of massive streaming data computing, the online learning method has attracted more and more researchers' attention. Online learning can be used in a dynamically changing learning environment, which sends training samples into the classifier one by one, and feeds back the results of the real category and the discriminant category to the classifier to complete the update of the classifier. It performs the training and identification process simultaneously, and can realize identification in real-time. The online learning method is good at capturing the trend of data, and can solve the problem of data different-distribution and real-time learning [6].

The classic online learning methods mainly include: First-order online learning, second-order online learning, budget quantity kernel method based online learning, and kernel approximation based online learning. The most commonly used first-order online learning method is the Passive Aggressive algorithm [7], which is a large-interval classifier with good performance in terms of stability and classification accuracy. Second-order online learning methods include Confidence Weighted algorithm [8], Soft Confidence Weighted algorithm [9], and Adaptive Regularization of Weight Vectors algorithm [10], which can mine the deep structure in the feature. The most commonly used framework of the budget quantity kernel method based online learning method is the Batch Stochastic Gradient Descent algorithm [11]. It refers to when the prediction is wrong, the support vector is added to the support vector set, and when the number of support vectors in the support vector set is greater than the budget quantity, the budgetary maintenance strategy is taken.

The kernel approximation based online learning methods include the Fourier Online Gradient Descent algorithm [12] and the Nyström Online Gradient Descent algorithm [13], which enables linear online learning in a new sample space derived from the kernel function approximation.

\section{Radar Emitter Identification under Transfer Learning and Online Learning}

This section first proposes a support vector machine model based on the TrAdaBoost method for the case where the target domain contains only a small number of labeled samples. Then, for the case of the target domain without labeled samples, the EM algorithm is used for transductive transfer learning. Finally, to overcome the problem of the poor timeliness of the above two methods, we propose a radar emitter identification method based on online learning.

\subsection{Support Vector Machine Model Based on the TrAdaBoost Method}

The TrAdaBoost method [14] is an inductive transfer learning method that involves two domains: The source domain $D_{s}$ and the target domain $D_{t}$. In the radar emitter identification task, the source domain $D_{s}$ refers to the known radar emitter data collected at ordinary times, and the target domain $D_{t}$ refers to the new radar emitter data collected by means of reconnaissance in wartime. The labeled samples in the source domain $D_{s}$ and a small number of labeled samples in the target domain $D_{t}$ are used as training sets. By continuously adjusting the weights of the samples, the data in the source domain $D_{s}$ that is not suitable for the target domain $D_{t}$ can be filtered out. In the process of training, if the labeled sample in the source domain $D_{s}$ is identified incorrectly, indicating that the correlation between the sample and the target domain $D_{t}$ is small, the weights of the sample are reduced, and in the subsequent training, the impact of the sample on the model will also be weakened. If the labeled sample in the target domain $D_{t}$ is identified incorrectly, the weights of the sample are increased, and the impact of the sample on the model will increase in later training. The principle of training is shown in Figure 1. 


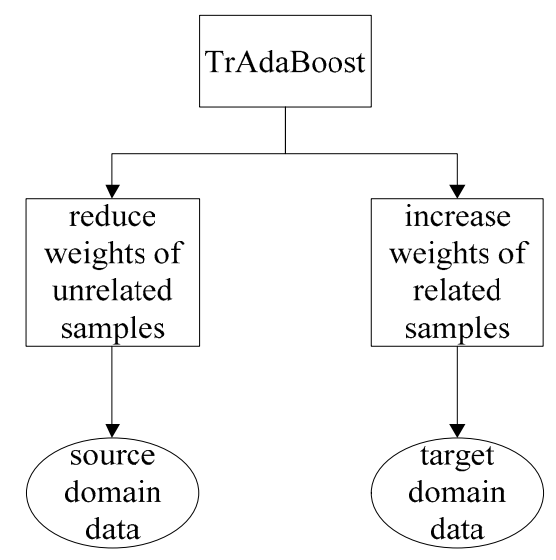

Figure 1. Training principle of the TrAdaBoost method.

The support vector machine model has the characteristics of simple structure and global optimization, and is good at solving small-sample and nonlinear problems. Therefore, this section chooses the support vector machine model as the base classifier to perform radar emitter identification. The TrAdaBoost method is used to train the base classifier support vector machine (SVM) model. The labeled samples in the source domain $D_{s}$ and a small number of labeled samples in the target domain $D_{t}$ are aggregated as the initial training set T. The unlabeled samples in the target domain $D_{t}$ are used as the test set $\mathrm{S}$. The specific flow is shown in Algorithm 1.

Algorithm 1. Support vector machine model based on the TrAdaBoost method

1. Initialize the weight vector $W^{1}=\left(w_{1}^{1}, \ldots, w_{\left|D_{s}\right|+\left|D_{t}\right|}^{1}\right)$, where

$$
w_{i}^{1}= \begin{cases}1 /\left|D_{s}\right|, & i=1, \ldots,\left|D_{s}\right| \\ 1 /\left|D_{t}\right|, & i=\left|D_{s}\right|+1, \ldots,\left|D_{s}\right|+\left|D_{t}\right|\end{cases}
$$

2. For $\mathrm{t}=1,2, \ldots, \mathrm{N}$

Set the weight distribution of the training set samples $P^{t}=\frac{W^{t}}{\sum_{i=1}^{\left|D_{s}\right|+\left|D_{t}\right|} w_{i}^{t}} ;$

Calculate the error rate of the SVM model hypothesis $h_{t}: X \rightarrow Y$ on the labeled samples in the source domain $D_{s}$, which is $\varepsilon_{t}=\sum_{i=1}^{\left|D_{t}\right|} \frac{w_{i}^{t}\left|h_{t}\left(x_{i}\right)-c\left(x_{i}\right)\right|}{\sum_{i=1}^{\left|D_{t}\right|} w_{i}^{t}}$;

If $h_{t}\left(x_{i}\right) \neq c\left(x_{i}\right)$

Reset the weight vector,

$$
w_{i}^{t+1}=\left\{\begin{array}{l}
w_{i}^{t}\left(1 /\left(1+\sqrt{2 \ln \left(\left|D_{s}\right| / N\right)}\right)\right)^{\left|h_{t}\left(x_{i}\right)-c\left(x_{i}\right)\right|},\left(x_{i}, y_{i}\right) \in D_{s} . \\
w_{i}^{t}\left(\varepsilon_{t} /\left(1-\varepsilon_{t}\right)\right)^{-\left|h_{t}\left(x_{i}\right)-c\left(x_{i}\right)\right|},\left(x_{i}, y_{i}\right) \in D_{t}
\end{array} .\right.
$$

$\mathrm{N}$ is the number of iterations.

End for

3. Get the final SVM model.

\subsection{Transductive Transfer Learning Based on EM Algorithm}

When there are labeled samples in the source domain $D_{s}$ and there are only unlabeled samples in the target domain $D_{t}$, the two domains face the same tasks, but their conditional probabilities $P(y \mid x)$ are still biased. The Expect Maximum algorithm (EM) [15] is good at finding the likelihood 
estimation value of the implicit parameters. Therefore, this section uses the EM algorithm to construct a transductive transfer learning framework. The specific flow is shown in Algorithm 2.

Algorithm 2. Transductive transfer learning based on EM algorithm

1. Learn on labeled samples in the source domain $D_{s}$, and get the initial hypothesis probability.

2. Assume that unlabeled samples in the target domain $D_{t}$ obey the data distribution $\Omega_{t}$.

For $\mathrm{t}=1,2, \ldots$, NExpect: Calculate the implied variable value according to the current hypothesis probability, and obtain the label information of the unlabeled samples in the target domain $D_{t}$ under the data distribution $\Omega_{t}$;

Maximum: Recalculate the new hypothesis based on the label information obtained above, and update the current hypothesis;

$\mathrm{N}$ is the number of iterations.

End for

3. Get the Optimal hypothesis $\Omega_{t}$.

The role of the EM algorithm is to be able to perform maximum likelihood estimation with implicit parameters, where the implicit parameters refer to the label information of the unlabeled samples in the target domain $D_{t}$. Therefore, the transductive transfer learning based on EM algorithm can realize the maximum likelihood estimation of the label information of the unlabeled samples in the target domain $D_{t}$ by means of the parameter estimation ability of the EM algorithm to obtain the optimal posterior hypothesis.

\subsection{Radar Emitter Identification Based on Online Learning}

The support vector machine model based on TrAdaBoost method and the transductive transfer learning method, based on the EM algorithm above, can solve the problem of knowledge transfer in different scenarios, but they still belong to offline learning models, which need the full set of training data for each training, so their real-time performance is poor. Therefore, this section proposes a radar emitter identification method, based on online learning, which can update the structure of the model in real-time to achieve fast and accurate identification.

The main idea of the online learning method is to construct a classifier using a subset of the training data instead of the full set. The measured parameters are $\mathbf{x}=\left[x_{1}, x_{2}, \ldots, x_{l}\right]$, and the unknown parameters to be estimated are $\omega=\sum_{i=1}^{l} \beta_{i} \phi\left(x_{i}\right)$, where $\beta_{i} \in R$ is a group of generic coefficients. By adding the definition of the kernel and the matrix $\mathrm{K},\|\omega\|^{2}$ can be written as $\sum_{i, j=1}^{l} \beta_{i} \beta_{j} \phi\left(x_{i}\right) \cdot \phi\left(x_{j}\right)=\sum_{i, j=1}^{l} \beta_{i} \beta_{j} K_{i j}$. Therefore, the optimization problem can be transformed into the following form:

$$
\begin{aligned}
& \underset{\beta, b}{\operatorname{argmin}} \frac{1}{2} \sum_{i, j=1}^{l} \beta_{i} \beta_{j} K_{i j}+C \sum_{i=1}^{l} \xi_{i}^{p} \\
& \text { s.t. } \quad y_{i}\left(\sum_{j=1}^{l} \beta_{i} K_{i j}+b\right) \geq 1-\xi_{i} \quad \forall i=1,2, \ldots, l
\end{aligned}
$$

where $C$ is error penalty factor, $\xi_{i}$ is slack variable, the value of $p$ is 1 or 2 , and $b \in R$.

The Lagrangian operator is calculated as follows:

$$
L_{P}^{\prime}=\sum_{i, j}^{l}\left(\frac{1}{2} \beta_{i}-\alpha_{i} y_{i}\right) \beta_{j} K_{i j}-\sum_{i=1}^{l} \alpha_{i}\left(b y_{i}-1+\xi_{i}\right)+\sum_{i=1}^{l} C \xi_{i}^{p}-\sum_{i=1}^{l} \mu_{i} \xi_{i}
$$


where $\alpha_{i}, \mu_{i} \geq 0$ are the 1-pair coefficients introduced.

Add Karush-Kuhn-Tucker conditions, and get

$$
\frac{\partial L_{P}^{\prime}}{\partial \beta_{i}}=\sum_{j=1}^{l}\left(\beta_{j}-\alpha_{j} y_{j}\right) K_{i j}=0, \quad \forall i=1,2, \ldots, l
$$

This section adopts the idea of one-against-one and uses the voting method to implement the decision of the model. The flow of learning and updating is shown in Figure 2.

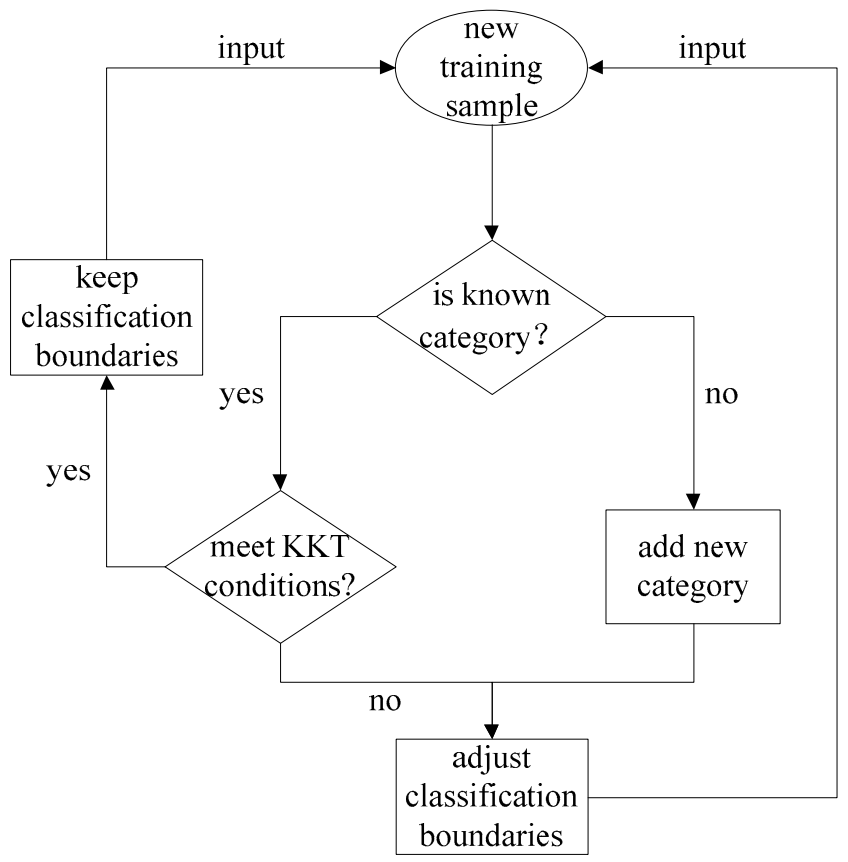

Figure 2. Flow of online learning and updating in radar emitter identification model.

When a new radar emitter arrives, if the emitter's category is known, then it is judged as to whether it meets the KKT conditions. If KKT conditions are met, it indicates that the emitter keeps the classification boundaries of the existing model, so it is not necessary to update the model. If the KKT conditions are not met, it indicates that new knowledge can be learned from the emitter to adjust the classification boundaries of the existing model, so the model can be updated. If the category of the emitter is unknown, then a new category needs to be added to the model and its classification boundaries are re-adjusted.

\section{Experiments}

\subsection{Experiment Settings}

\subsubsection{Experiment Environment}

We built the simulation experiment development environment of Windows7 + Matlab2017b + Libsvm3.22, where Libsvm3.22 was used to implement the SVM model as the base classifier. Its kernel function was based on the radial basis function $\exp \left(-\frac{\left|x-x_{i}\right|^{2}}{\sigma^{2}}\right)$. On this basis, we used Matlab to realize the transfer learning and online learning methods in this paper.

\subsubsection{Experiment Data}

We use the characteristic parameters such as pulse amplitude (PA), carrier frequency (CF), pulse width (PW), pulse repetition interval (PRI), and angle of arrival (AOA) to represent the emitter 
data, and select three working modes of search, tracking, and guidance as the category labels of the emitter. Three-thousand signal samples are generated using the above five pulse descriptors for each working mode, and a total of 9000 signal samples are the source domain data. Using the above five pulse descriptors for each working mode, 3000 signal samples are generated with different distributions, and a total of 9000 signal samples are the target domain data. In addition, in order to verify the performance of the proposed method, we add different degrees of noise to the experimental data. The details of the experiment data are shown in Tables 1 and 2. Table 1 shows the conventional radar characteristic parameters in the source domain, while Table 2 shows the unknown radar characteristic parameters in the target domain.

Table 1. Conventional radar characteristic parameters.

\begin{tabular}{cccccc}
\hline Working Mode & PA & CF/MHz & PW/ $\mu$ s & PRI $/ \mu$ s & AOA/ $^{\circ}$ \\
\hline search & {$[16,20]$} & {$[3121,3333]$} & {$[7.1,7.2]$} & {$[800,860]$} & {$[66,68]$} \\
tracking & {$[6,16]$} & {$[2019,2020]$} & {$[1.1,1.3]$} & {$[400,550]$} & {$[46,48]$} \\
guidance & {$[2,12]$} & {$[2150,2250]$} & {$[0.3,0.5]$} & {$[300,400]$} & {$[62,64]$} \\
\hline
\end{tabular}

Table 2. Unknown radar characteristic parameters.

\begin{tabular}{cccccc}
\hline Working Mode & PA & CF/MHz & PW/ $\boldsymbol{\mu s}$ & PRI $/ \mu$ s & AOA $^{\circ}$ \\
\hline unknown mode1 & {$[22,30]$} & {$[2850,3098]$} & {$[4.5,4.6]$} & {$[620,680]$} & {$[45,47]$} \\
unknown mode2 & {$[11,13]$} & {$[2550,2551]$} & {$[0.4,0.6]$} & {$[700,760]$} & {$[25,27]$} \\
unknown mode3 & {$[4,7]$} & {$[2748,2758]$} & {$[0.5,0.7]$} & {$[220,300]$} & {$[50,52]$} \\
\hline
\end{tabular}

The mean values and standard deviations after normalization of the source domain data and the target domain data are significantly different, so they no longer satisfy the assumption of the same distribution. The details are shown in Table 3. For the transfer learning scenario with only a small number of labeled samples in the target domain, we set $15 \%$ of the samples in the target domain to be labeled samples. For the transfer learning scenario with no labeled samples in the target domain, we set all samples in the target domain to be unlabeled samples. For the online learning scenario, we set $15 \%$ of the samples in the target domain as the training data, and randomly select $50 \%$ of the samples as fixed samples, and add the remaining $50 \%$ of the samples as new samples to the training set in order. Through the above construction of the experiment data, it can be used to verify the transfer learning and online learning methods.

Table 3. Distribution difference after normalization of the source domain data and the target domain data.

\begin{tabular}{ccccc}
\hline & \multicolumn{2}{c}{ Source Domain Data } & \multicolumn{2}{c}{ Target Domain Data } \\
\hline Characteristic Parameter & Mean Value & Standard Deviation & Mean Value & Standard Deviation \\
\hline PA & 0.72 & 0.21 & 0.86 & 0.09 \\
$\mathrm{CF} / \mathrm{MHz}$ & 0.81 & 0.12 & 0.61 & 0.26 \\
$\mathrm{PW} / \mu \mathrm{s}$ & 0.76 & 0.32 & 0.44 & 0.13 \\
$\mathrm{PRI} / \mu \mathrm{S}$ & 0.86 & 0.24 & 0.54 & 0.09 \\
$\mathrm{AOA} /{ }^{\circ}$ & 0.59 & 0.03 & 0.78 & 0.16 \\
\hline
\end{tabular}

In the actual application process, when dealing with real radar data, we must first collect, process, and sort the radar signals to get the pulse described words. The goal of this article is to identify the system or behavior of radar emitters, so using only the pulse described words can meet the needs of this article. However, if the modulation pattern of radar signals needs to be identified, we must use more information in the radar waveforms. In addition, for fast time-varying scenarios, we must improve the signal processing efficiency of radio frequency front modules as much as possible to ensure the effective use of the method in this article, which will be an urgent problem in the future. 


\subsubsection{Experiment Scenario}

In the following, we will set up three different experiment scenarios to verify the effectiveness of the proposed methods.

Scenario 1: In the scenario, there are only a few labeled samples in the target domain. It means that some samples encountered during the actual application are the same as the original trained samples, so we can easily obtain the labels of these samples.

Scenario 2: In the scenario, the target domain does not contain labeled samples. It means that all the samples encountered in the actual application process are very different from the original trained samples, so it is difficult for us to obtain the labels of these samples.

Scenario 3: In the scenario, we constantly encounter samples that are significantly different from the original trained samples, so we must immediately update our model to identify these samples quickly and accurately.

\subsection{Contrast Experiments under Transfer Learning}

For the transfer learning scenario where there are only a few labeled samples in the target domain, we set up the following three sets of contrast experiments:

Experiment 1: We train the base classifier SVM model on the labeled samples in the target domain, and then perform the identification on the unlabeled samples in the target domain, which is denoted as SVM1_TransferOne.

Experiment 2: We train the base classifier SVM model on all labeled samples in the source domain and target domain, and then perform the identification on the unlabeled samples in the target domain, which is denoted as SVM2_TransferOne.

Experiment 3: We train the SVM model based on the TrAdaBoost method on all labeled samples in the source domain and target domain, and then perform the identification on the unlabeled samples in the target domain, which is denoted as TrAdaBoost_TransferOne.

The results of the experiment are shown in Figure 3. The identification accuracy of SVM1_TransferOne in the measurement error range of $15 \%$ is only $52.6 \%$, which is not up to the actual application standard. The main reason is that the number of labeled samples in the target domain is small, and the SVM model cannot be fully trained. Compared to SVM1_TransferOne, SVM2_TransferOne uses more training samples, but the identification accuracy is lower, mainly because the source domain data and the target domain data do not satisfy the same-distribution hypothesis, and the SVM model cannot be effectively trained. TrAdaBoost_TransferOne achieves the highest identification accuracy, which is up to $74.5 \%$ in the measurement error range of $15 \%$, indicating that the TrAdaBoost-based method can use the limited labeled samples in the target domain to filter more samples of use to the target domain from the source domain. This proves the effectiveness of the method.

For the transfer learning scenario where the target domain does not contain labeled samples, we set up the following two sets of contrast experiments:

Experiment 4: We train the base classifier SVM model on the labeled samples in the source domain, and then perform the identification on the unlabeled samples in the target domain, which is denoted as SVM_TransferTwo.

Experiment 5: We use the transductive transfer learning based on EM algorithm to train on the labeled samples in the source domain and the unlabeled samples in the target domain, and then perform the identification on the unlabeled samples in the target domain, which is denoted as EM_TransferTwo. 


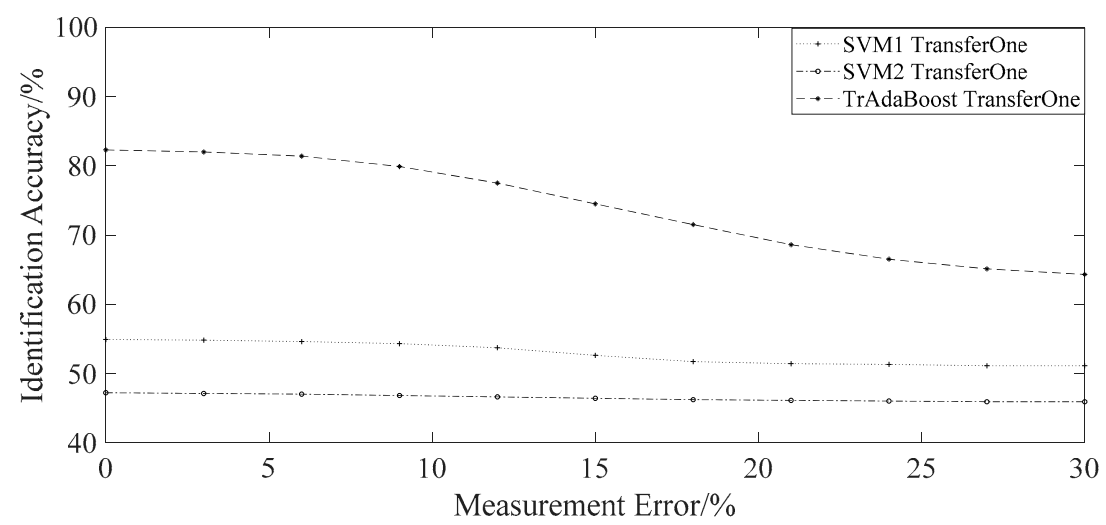

Figure 3. Contrast experiment results in the case where the target domain contains only a small number of labeled samples.

The experiment results are shown in Figure 4. The identification accuracy obtained by SVM_TransferTwo is very low, at only $46.1 \%$ in the measurement error range of $15 \%$. The main reason is that the source domain data and the target domain data do not satisfy the same-distribution hypothesis, but the target domain does not contain labeled samples, so the TrAdaBoost-based method is not applicable. Compared to SVM_TransferTwo, the identification accuracy of EM_TransferTwo is significantly improved, up to $69.9 \%$ in the measurement error range of $15 \%$. This indicates that the transductive transfer learning based on EM algorithm can realize the transfer of knowledge in the case where the target domain does not contain labeled samples, and proves the effectiveness of the method.

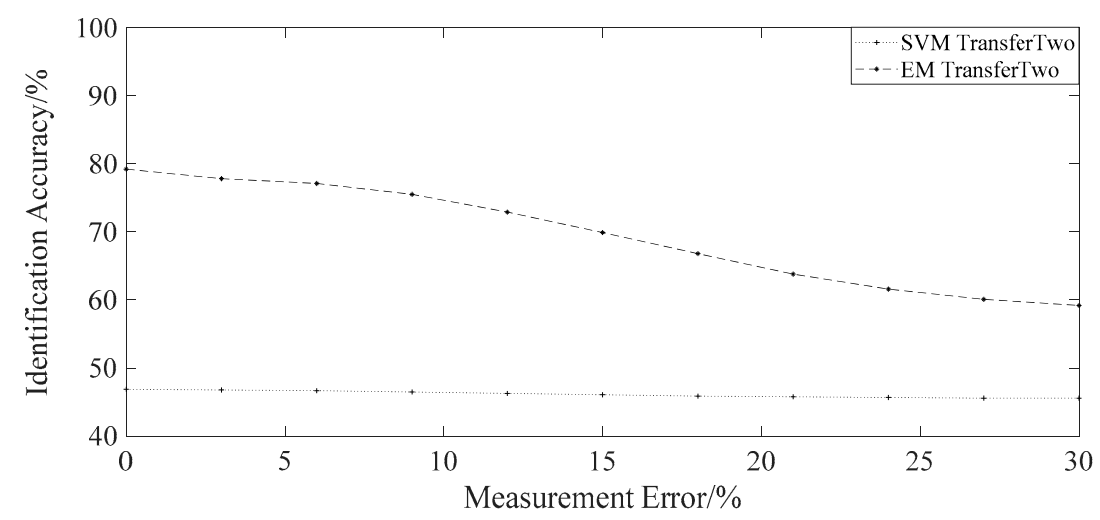

Figure 4. Contrast experiment results in the case where the target domain does not contain labeled samples.

In summary, in the scenario where there are only a few labeled samples in the target domain and the target domain does not contain labeled samples, the transfer learning method is significantly better than the traditional method. It indicates that the transfer learning method can effectively utilize a large number of labeled samples in the source domain, extract useful knowledge, and reduce the number of wasted labeled samples. In addition, it can overcome the problem that training data and testing data do not satisfy the same-distribution hypothesis, resulting in low identification accuracy.

\subsection{Contrast Experiments under Online Learning}

For the online learning scenario, we set up the following two sets of contrast experiments:

Experiment 6: We train the base classifier SVM model on the training data in the target domain, and then perform the identification on the testing data in the target domain, which is denoted as SVM_Online. 
Experiment 7: We use the radar emitter identification method based on online learning to train the training data in the target domain, and then perform the identification on the testing data in the target domain, which is denoted as REI_Online.

SVM_Online and REI_Online are repeated 20 times to average the results, and the running time and identification accuracy are shown in Figures 5 and 6. From the perspective of the model running time, SVM_Online requires longer training time, and the longest training time is $189 \mathrm{~s}$. The main reason is that the previous training results cannot be used effectively when only using the base classifier SVM model. When the new samples are added to the training set, the training has to be restarted. When the number of training samples increases, the training time will increase linearly. However, REI_Online uses the online learning method, and the time required is almost constant. Its average running time is only $10.5 \mathrm{~s}$. From the perspective of the identification accuracy, with the continuous addition of new samples, the identification accuracy obtained by REI_Online will continue to increase, and will converge towards SVM_Online's, and the final identification accuracy is slightly lower than SVM_Online's. The main reason is that the number of training samples is small at the beginning of training, which results in low identification accuracy. When the number of training samples increases, the online classifier will be improved and the identification accuracy will be improved accordingly. However, SVM_Online uses the complete training set each time, so it can obtain the highest identification accuracy. In addition, due to the limited number of training samples used, SVM_Online and REI_Online do not have the identification accuracy of more than $60 \%$. In summary, the radar emitter identification method based on online learning can obtain the identification accuracy similar to the traditional method, but it can greatly shorten the running time. This proves the effectiveness of the method.

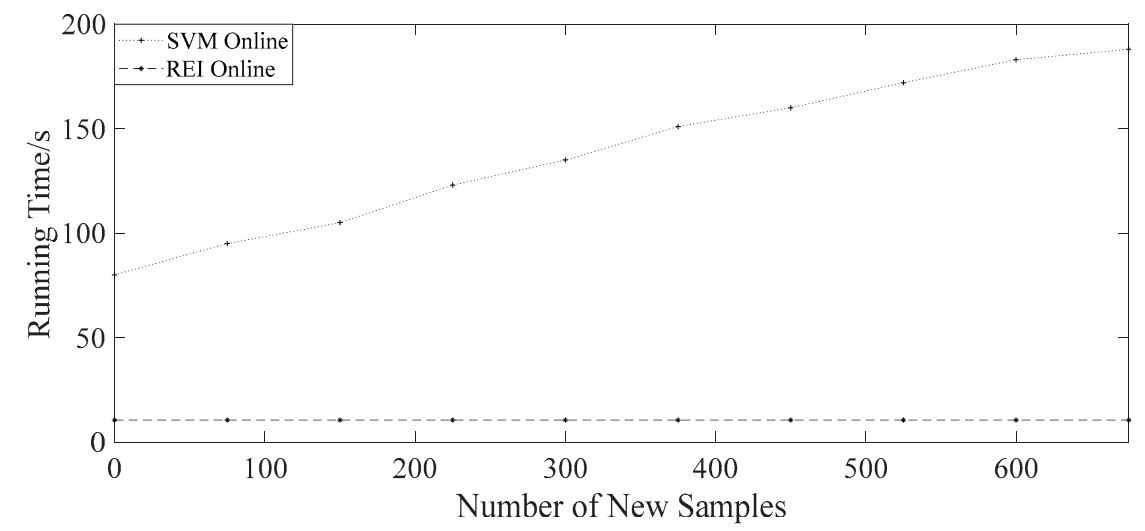

Figure 5. Running time of contrast experiment in the online learning scenario.

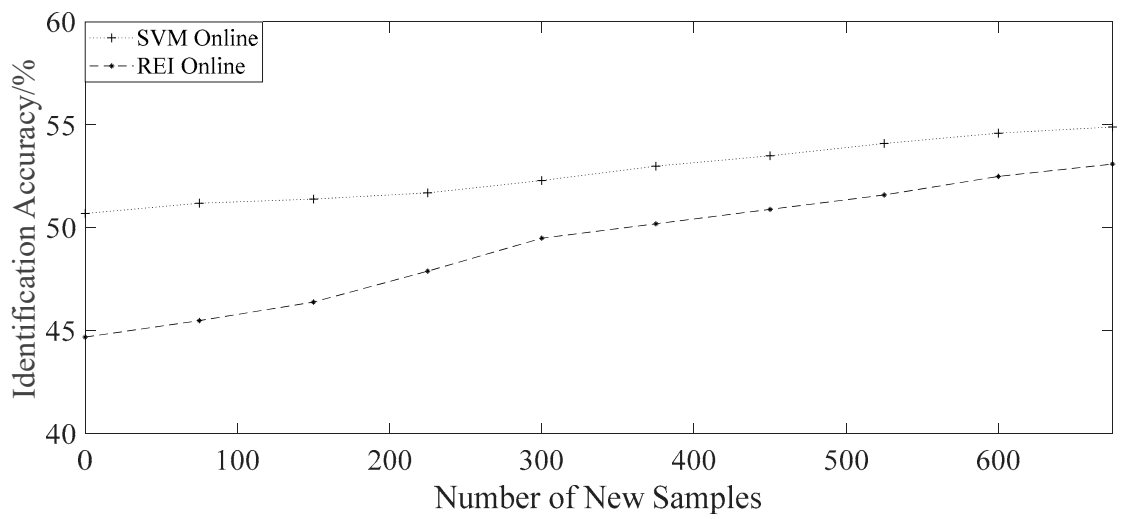

Figure 6. Identification accuracy of contrast experiment in the online learning scenario. 


\subsection{A Combination of Transfer Learning Method and Online Learning Method}

In the actual combat background, the above REI_Online can greatly shorten the running time, but its identification accuracy cannot reach the standard of practical application. The main reason is that it is difficult to obtain enough labeled data in the target domain. Therefore, in response to the above problem, when the target domain contains only a small number of labeled samples and new samples are continuously added for online learning, we combine the transfer learning method with the online learning method, which can make comprehensive use of useful information in the source domain data and the target domain data. We use the combination method to perform the identification on the testing data in the target domain, which is denoted as TrAdaBoost_Online. The results of the experiment are shown in Figures 7 and 8. The running time of TrAdaBoost_Online is only 5.3\% of the above TrAdaBoost_TransferOne, with an average of $23 \mathrm{~s}$, which is significantly reduced. The identification accuracy of TrAdaBoost_Online almost reaches the level of TrAdaBoost_TransferOne, and reaches $73.5 \%$ in the measurement error range of $15 \%$, which has good noise adaptability.

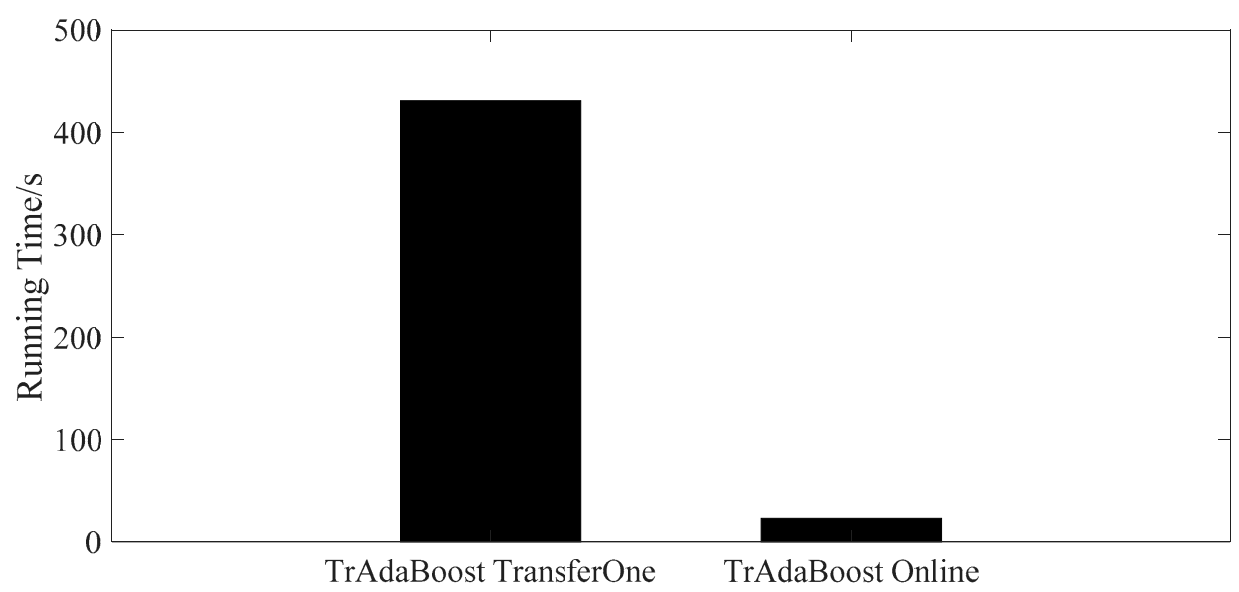

Figure 7. Running time of the combination method.

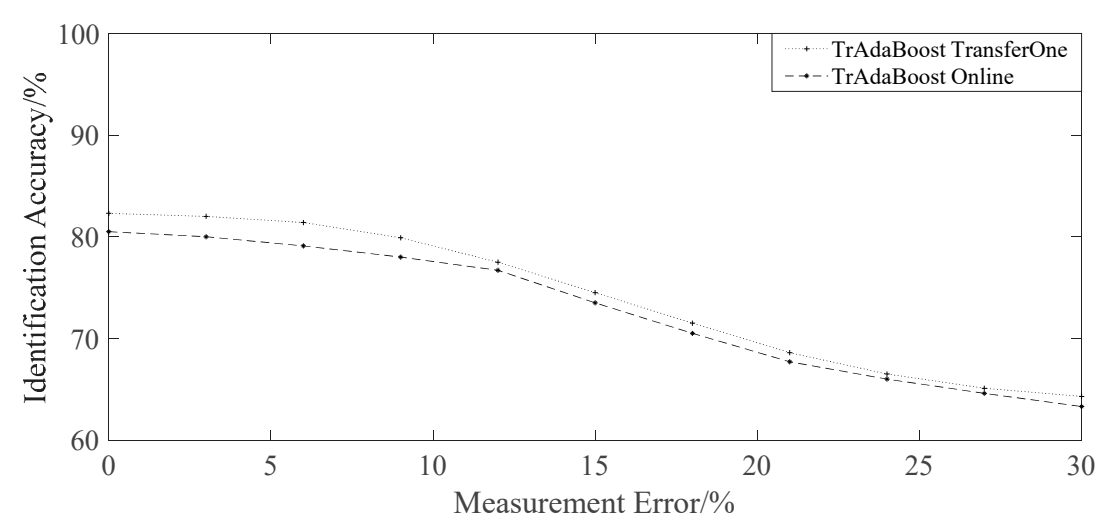

Figure 8. Identification accuracy of the combination method.

In the first stage, we use the detected radar emitter data to train our model. However, in the stage of practical application, the situation is often changing rapidly, and there will be many radar emitters that have not been seen in the past. Satisfactory results cannot be achieved by using the original model, so we can use the method based on online learning to update the model in real-time in order to meet the actual needs of the battlefield. In addition, we have verified the effectiveness of combining the transfer learning algorithms with the online learning algorithm in the radar emitter identification task. Therefore, the method based on online learning can be used to improve the speed of the transfer learning algorithms proposed in this paper. 


\section{Conclusions}

In this paper, the ideas of transfer learning and online learning are applied to the field of radar emitter identification. For two different transfer learning scenarios, we propose the support vector machine model based on the TrAdaBoost method and transductive transfer learning based on the EM algorithm. Finally, in order to overcome the problem of the poor timeliness of the above two methods, we propose a radar emitter identification method based on online learning. Simulation experiments show that the combination of transfer learning and online learning methods can obtain higher identification accuracy and good timeliness, and can be applied in the actual combat background. The next step is to optimize the combination model and to improve the identification accuracy to enhance its practicability.

Author Contributions: Conceptualization, G.W.; Investigation, R.W.; Resources, Y.C.; Supervision, X.X.; Validation, H.H.; Writing - review \& editing, Y.F. All authors have read and agreed to the published version of the manuscript.

Funding: This research received no external funding.

Conflicts of Interest: The authors declare no conflict of interest.

\section{References}

1. Hugh, G. ELINT: The Interception and Analysis of Radar Signals R.G. Wiley Artech House, 46 Gillingham Street, London, SW1V 1AH. 2006. 451pp. Illustrated. £82.00. ISBN 1-58053-925-4. Aeronaut. J. 2007, 111, 280.

2. Lei, W.; Ji, H. Optimizing zero-slice feature of ambiguity function for radar emitter identification. In Proceedings of the 7th International Conference on Information, Communications and Signal Processing (ICICS), Macau, China, 8-10 December 2009.

3. Bian, M.; Wang, S.; Hu, W.; Ma, L.; Liu, S.; An, D. Radiation source and scattering characteristics analysis of space-borne terahertz cloud radar. J. Terahertz Sci. Electron. Inf. Technol. 2015, 13, 712-717. (In Chinese)

4. Nguyen, M.N.; Rajapakse, J.C. Two-Stage support vector machines for protein secondary structure prediction. Neural Parallel Sci. Comput. 2003, 11,1-18.

5. Pan, S.J.; Yang, Q. A Survey on Transfer Learning. IEEE Trans. Knowl. Data Eng. 2009, 22, 1345-1359. [CrossRef]

6. Shalevshwartz, S. Online Learning and Online Convex Optimization. Found. Trends Mach. Learn. 2012, 4, 107-194. [CrossRef]

7. Lu, J.; Zhao, P.; Hoi, S.C.H. Online Passive-Aggressive Active learning. Mach. Learn. 2016, 103, $141-183$. [CrossRef]

8. Wang, J.; Zhao, P.; Hoi, S.C.H. Exact soft confidence-weighted learning. arXiv 2012, arXiv:1206.4612.

9. Bistarelli, S.; Gennari, R.; Rossi, F. General Properties and Termination Conditions for Soft Constraint Propagation. Constraints 2003, 8, 79-97. [CrossRef]

10. Crammer, K.; Kulesza, A.; Dredze, M. Adaptive regularization of weight vectors. Mach. Learn. 2013, 91, 155-187. [CrossRef]

11. Tsuruoka, Y.; Tsujii, J.; Ananiadou, S. Stochastic Gradient Descent Training for L1-regularized Log-linear Models with Cumulative Penalty. In Proceedings of the Joint Conference of the 47th Annual Meeting of the ACL and the 4th International Joint Conference on Natural Language Processing of the AFNLP, Suntec, Singapore, 2-7 August 2009; Association for Computational Linguistics: Stroudsburg, PA, USA, 2009; pp. 477-485.

12. Lu, J.; Hoi, S.C.H.; Wang, J.; Zhao, P.; Liu, Z.Y. Large Scale Online Kernel Learning. J. Mach. Learn. Res. 2016, $17,1613-1655$.

13. Peter, L.; Bartlett, E.H.; Alexander, R. Adaptive Online Gradient Descent. In Advances in Neural Information Processing Systems, Proceedings of the Twenty-First Annual Conference on Neural Information Processing Systems, Vancouver, BC, Canada, 3-6 December 2007; Curran Associates Inc.: Red Hook, NY, USA, 2007. 
14. Zhang, Q.; Li, H.; Zhang, Y.; Li, M. Instance Transfer Learning with Multisource Dynamic TrAdaBoost. Sci. World J. 2014, 2014, 282747. [CrossRef]

15. Papaconstadopoulos, P.; Levesque, I.R.; Maglieri, R.; Seuntjens, J. Direct reconstruction of the source intensity distribution of a clinical linear accelerator using a maximum likelihood expectation maximization algorithm. Phys. Med. Biol. 2016, 61, 1078-1094. [CrossRef] 